\title{
Internal Morphology of Diamonds from Pipe Udachnaya According to the Data of Luminescence Tomography Method
}

Mironov, V.P.

YaGEER\&D CNIGRI, ALROSA Co. Ltd. Chernyshevsky Sh. 7, Mirny, 678170, Sakha (Yakutia), Russia.

Under the change of crystallization conditions (temperature, pressure or chemical composition of environment) as a rule there occurs alteration of the mechanism of the crystal's growth, which causes alteration of the form of its growth. If early growth forms differentiate from each other by physical features, then there is an opportunity to detect them by a number of methods. The methods applied for detection of growth mechanisms were discussed in detail in recent review of Lang, A.R. (Lang, 1993). Application of those methods for solving geological tasks is complicating, and sometimes even impossible, for most of them require cutting of samples. This brings to limitation of massive investigations and does not allow to make use of statistical methods.

The method of luminescence tomography (LT) is relatively simple, does not require cutting of crystals and does not take much time for a detection. That is why there is an opportunity to study hundreds of crystals and receive statistically reliable regularities (Mironov and Izenev, 1992). The essence of the method lies in selective excitation of luminescence of crystals' sections which is equivalent to observation of a plate cut out by this section. The LT method made it possible to determine regularities of Yakutian diamonds' growth (Mironov and Antonuk, 1994), four consecutively replacing each other stages of crystallization were revealed (Mironov, 1995), as well as distinctions in internal morphology of crystals from high-diamondiferous and low-diamondiferous pipes of Malo-Botuobinsky kimberlite field were discovered (Mironov, 1996).

The present work contains results of investigations of internal morphology of diamonds from pipe Udachnaya in comparison with the internal morphology of earlier investigated (Mironov, 1995) diamonds from pipe Mir. The investigated selection consisted of 1000 crystals of $1-4 \mathrm{~mm}$ in size, mainly octahedrons and their twins according to the spinel law, as the most convenient for investigation by LT method. In the course of investigation each crystal received numerical code indicating stages of crystallization during which the growth of a definite individual and succession of their replacement took place. It should be reminded that the earliest ones (the first stage of crystallization) are correlated with phantom crystals with green or yellow-green luminescence, the second stage is correlated with the crystals and zones with blue luminescence, the third stage - with the crystals and phantom crystals with orange luminescence and the fourth, the latest stage, is correlated with nonluminescent crystals and or their nonluminescent external zones (Mironov and Antonuk, 1994). In accordance with this the 134 code means that the crystal has the central part with green luminescence, which is surrounded by a coat with orange 
luminescence and then by nonluminescent external zone, and it grew consecutively at the first, the third and the fourth stages of crystallization. Correspondingly code 12 means that the crystal consists of the phantom crystal with green luminescence surrounded by a coat with blue luminescence.

The code consisting of one numeral means that the crystal is homogeneous and it grew only at one of the stages, with the number of the stage corresponding to the colour of luminescence.

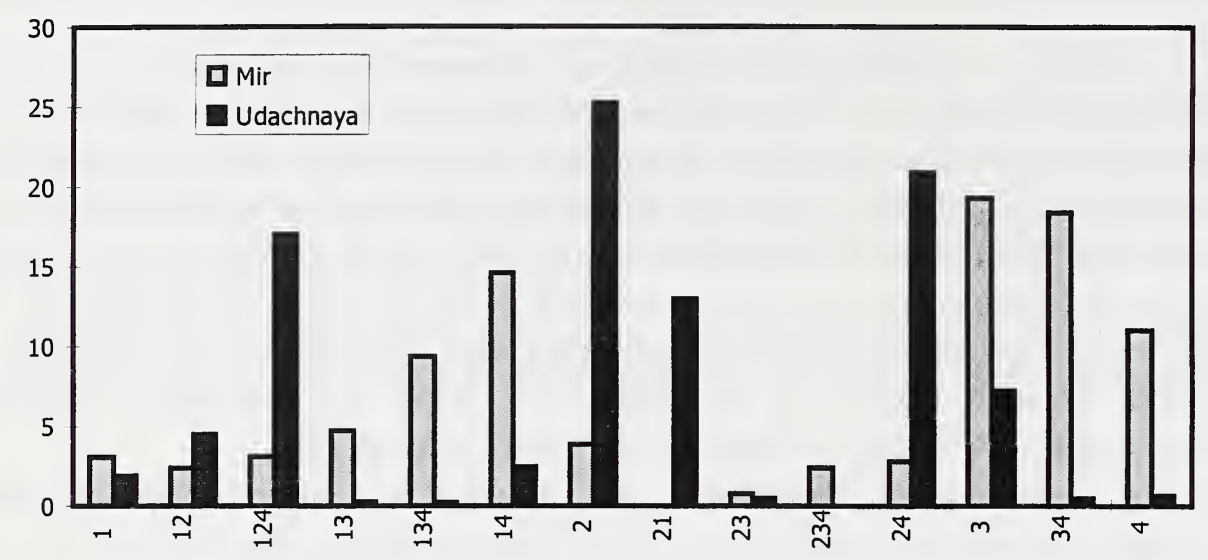

\section{Distribution of diamonds from pipes Mir and Udachnaya according to the type of morphology}

Crystals with similar internal structure, that is, with identical code, were united into groups and the frequency of occurrence of such crystals in total selection was calculated. Distribution of crystals according to the type of structure is given in the table and in the histogram. Here for comparison the results of similar investigations for pipe Mir are shown, as well.

In the selection of diamonds from pipe Udachnaya $34.9 \%$ of crystals have homogeneous distribution of luminescence through the bulk of a sample, that is, their growth occured only at one of the stages of crystallization, with $25.2 \%$ from the total number of diamonds growing only at the second stage of crystallization. The crystals which grew at two stages of crystallization compose $29.7 \%$, with $20.9 \%$ of all crystals growing at the second and then at the fourth stages and correspondingly consisting of internal phantom crystal with blue luminescence in nonluminescent coat. Phantom crystals with blue luminescence are well outlined and may have the shape from well cut octahedrons to a rounded shape.

Crystals consisting of three zones consequently having grown at three stages of crystallization compose $30.8 \%$, with the growth of more than half of them (17.7\%) occurring in succession at the first, the second and the fourth stages, in the result of which the colour of their growth zones changes in the following succession: green - blue nonluminescent zone. The central phantom crystal with yellow-green luminescence may be of two types. 1. Well outlined with bright yellow-green luminescence and having the 
shape similar to cubes or their fragments. 2. Phantom crystals having less bright green luminescence, distribution of which points to simultaneous growth of a crystal by facets of a "cube" and octahedron. Luminescence is concentrated in pyramids of a "cube". Pyramids of an octahedron in these phantom crystals are nonluminescent, sometimes with blue luminescence. Both types of phantom crystals are surrounded by a coat with blue luminescence.

Groups of crystals with inversion of luminescence colours 214, 212, 2124 should be noted especially. These groups of crystals are not accidental, since total frequency of their occurrence compose about $13 \%$. Such crystals practically do not occur in highdiamondiferous sources of Malo-Botuobinsky kimberlite field.

Analyzing the table and histogram one should note that internal morphology of diamonds from pipe Udachnaya sufficiently differ from the same for the diamonds from pipe Mir, since in the investigated selection of diamonds from pipe Udachnaya the greatest frequency of occurrence belongs to nontypical for pipe Mir homogeneous crystals with blue luminescence $(25.9 \%)$, crystals consisting of a phantom crystal with blue luminescence in nonluminescent shell (21\%) and crystals containing phantom crystal with green luminescence, surrounded by blue and nonluminescent shells (17\%). On the other hand among the crystals from pipe Udachnaya there are practically no typical for pipe Mir (32\%) crystals consisting of orange phantom crystal in nonluminescent shell.

Thus, selections from pipes Mir and Udachnaya sufficiently differ in distribution according to the types of internal morphology, that may serve as indication pointing to belonging of the selection to this or that kimberlite pipe. Since statistical regularities of alteration of internal morphology of crystals are the result of change of crystallizational conditions, then justified is the conclusion about essential distinctions in conditions of crystallization of diamonds from pipes Mir and Udachnaya - the most important kimberlite pipes of Malo-Botuobinsky and Daldyn-Alakit kimberlite fields.

\section{References}

Lang, A.R., 1993, Topographic methods for studying defects in diamonds: Diamond and related materials, \# 2.

Mironov, V.P. and Izenev, V.N., 1992, Method of sorting of transparent crystals:Rus. Pat. \# 1830225.

Mironov, V.P. and Antonuk, B.P., 1994, Regularities of diamond growth from Yakutia: Archive of Mineralogy, \# 50 (2).

Mironov, V.P., 1995, Growth steps of diamonds from Yakutia: Proc. of The 6th IKC, Novosibirsk.

Mironov, V.P., 1996, Internal structure of diamonds: applied aspects.: Proc. of The 4 th International Congress of Applied Mineralogy, Warsaw. 